\title{
40 Jahre Immunglobulin E
}

mmunoglobulin E: A New Class of Human Immunoglobulin - das war der Titel eines vor 40 Jahren in den wichtigsten immunologischen und allergologischen Zeitschriften veröffentlichten Beitrags der Autoren Bennich, Ishizaka, Johansson, Rowe, Stanworth und Terry aus Schweden, USA, der Schweiz und England. Damit setzten diese Arbeitsgruppen den Schlussstrich unter einer mehrjährigen Erkenntnisphase, in der die Forscher auf getrennten Wegen schließlich zur gemeinsamen Gewissheit gelangten, eine neue Immunglobulinklasse entdeckt zu haben. Im Februar 1968 wurde dieser krönende Abschluss beim WHO International Reference Centre for Immunoglobulins in Lausanne erreicht.

Kimishige und Teruko Ishizaka in Denver hatten zuvor bereits im Jahr 1966 in mehreren experi„Die Forscher kamen auf mentellen Arbeiten getrennten Wegen schließlich beschrieben, dass es zur gemeinsamen Erkenntnis, sich bei den als „Redass sie eine neue Immun- agine" bezeichneten

globulinklasse entdeckt Antikörpern um eine hatten." Immunglobulinklasse handelte, die nicht zu den bekannten Immunglobulinklassen A, M, G oder D gehörte. Sie nannten diese „, $\gamma \mathrm{E}-\mathrm{Globu}-$ lin".

Gunnar Johansson und Hans Bennich aus Uppsala beschrieben im darauf folgenden Jahr ein atypisches Myelomprotein, das ebenfalls nicht zu den bekannten Immunglobulinen gehörte und das sie „Myelom-IgND“ nannten. Bereits 1967 tauschten die beiden Arbeitsgruppen spezifische Antiseren gegen die neuentdeckten Immunglobuline aus und

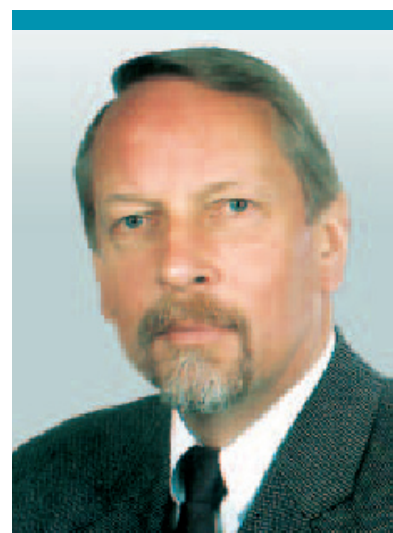

Prof. Dr. Gerhard SchultzeWerninghaus, Abteilung für Pneumologie, Allergologie und Schlafmedizin,

BG-Kliniken Bergmannsheil

- Universitätsklinik, Bochum

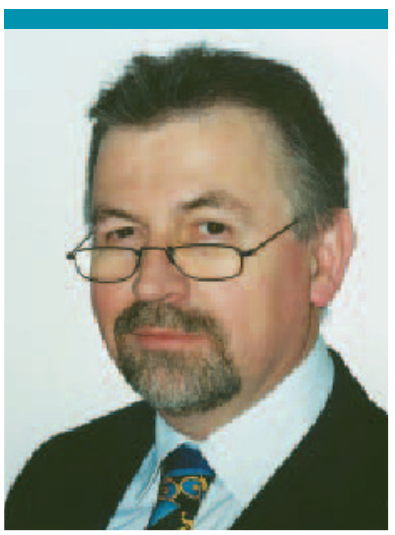

Prof. Dr. Bernhard Przybilla, Klinik und Poliklinik für Dermatologie und Allergologie, Klinikum der Ludwig-MaximiliansUniversität, München stellten die Übereinstimmung ihrer Entdeckungen fest.

Hilfreich bei dieser raschen Entwicklung war die gleichzeitige Entwicklung des Radioallergosorbent-Tests (RAST) durch Leif Wide in Uppsala mit Unterstützung durch Pharmacia vor Ort. Die Erstpublikation des Tests erfolgte 1967, zusammen mit Johansson und Bennich. Pharmacia brachte 1971 den RIST zur Bestimmung des Gesamt-IgE und 1974 den RAST zur Bestimmung des allergenspezifischen IgE auf den Markt. Damit war der Beginn einer neuen Ära der Allergologie innerhalb weniger Jahre vollzogen, an den in diesem Editorial erinnert werden soll.

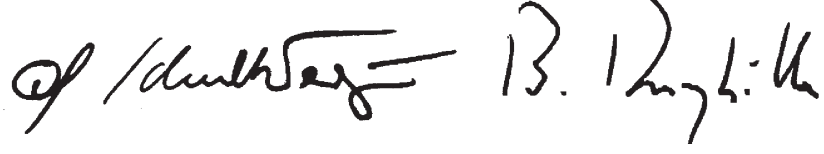

Prof. Dr. G. Schultze-Werninghaus Prof. Dr. B. Przybilla 